\title{
Complexity in a spatially uniform continuum fault model
}

\author{
Bruce E. Shaw \\ Iamont-Doherty Earth Observatory, Columbia University, Palisades, New York
}

Abstract Recently, Rice [1993] pointed out that, up to now, the self-organizing models which have produced complex nonperiodic sequences of events have all been sensitive to the spatial discretization used, and thus did not have a well defined continuum limit. He went on the suggest that spatial nonuniformity or "inherent discreteness" may be a necessary ingredient in allowing the complexity to develop in these systems. In this paper, I present a counterexample to this suggestion: a spatially uniform model with a well defined continuum limit is shown to give rise to complex nonperiodic sequences. The complexity arises in the deterministic model from inertial dynamics with a velocity-weakening frictional instability, with the instability being stabilized at short lengthscales by a viscous term. The numerical results are shown to be independent of the spatial discretization for discretizations small compared to the viscous lengthscale. Furthermore, the cualitative features of the complexity produced are seen to be invariant with respect to two very different types of small scale cutoffs, implying a universality of the results with respect to the details of the small scale cutoff.

\section{Introduction}

Earthquakes are complex in many ways. Where they occur, when they occur, how big they are, and what kind of shaking they produce, are just some of the complicated features we would like to understand. In trying to develop models that displayed the richness and complexity exhibited by earthquakes, seismologists were led to propose models with spatially inhomogeneous properties. The general belief was that material inhomogeneities were essential to generate the observed complexity. Recently, this point of view has been challenged by a class of models in which complexity arises along a uniform fault from the self-organization of repeated ruptures when inertial effects and velocity-weakening friction are included [Carlson and Langer, 1989]. In contrast, cuasistatic models which neglect inertial dynamical terms seem to recpuire some degree of heterogeneity in either driving or material properties, to produce complex sequences [Bak, Tang, and Wiesenfeld, 1987; Xu, Bergersen, and Chen, 1992; Cowie, Vanneste, and Sornette, 1093]. Real faults are, of course, spatially inhomogeneous, showing geometrical irregularities- fractal fault traces, steps, and bends- as well as frictional variations. The question is whether it is these fixed irregularities (which, while varying on a groological timescale, are essentially unchanging over the timescale on which the complexity is occurring) which are the dominant control of the complexity seen, or whether the dynamical fields which evolve during the earthcluake cycle itself- the stresses and strains- are the dominant control. It is clear that models where the dominant control is fixed inhomogeneities can show sufficient complexity to mimic the observations. The disadvantage of these models is that, what determines the distributions of inhomogeneities remains unexplained, and how one might measure the corresponding distributions for the real system remains unclear. The hope would be that there might be properties of these systems that are, though, somehow universal, and thus are independent of the distributions of inhomogeneities. The ability to circumvent this whole issue is a major reason why the spatially uniform models, which achieve complexity through dynamical instabilities, appear so attractive. For the spatially uniform self-organizing models, the question of whether they can show

\section{Copyright 1994 by the American Geophysical Union.}

Paper number 94GL01685

0094-8534/94/94GL-01685\$03.00 sufficient complexity in the case relevant to real earthquakes- fully dynamic three dimensional elastic interactions- is still unanswered. The main thing standing in the way of answering this question is the numerical costliness of dynamic elasticity. Typically, then, models neglect either the true dynamic aspects or the long range elastic aspects, and study a reduced less costly model. Thus the current situation regarding the self-organizing models is that there are hints that they may work to give sufficient complexity in the full case of interest, but no clear answers. Of course, even if they did give sufficient complexity, the Earth might still be in a regime where the fixed inhomogeneities were dominant. As we learn more about what the self-organizing models can and cannot do, though, the choice between the two alternatives should become clearer

In a recent paper, Rice [1993] has raised a serious concern regarding whether the self-organizing explanation can give rise to complexit.y on a truly spatially uniform fault. He points out that, up to now, all of the results from the self-organizing models have been sensitive to the spatial discretizations used. He further comments on results from his numerical model, which involves the quasistatic evolution of three dimensional elastic interactions, and which contains no inertial term. Again, the fully dynamic case is too costly to study; he decides to make the tradeoff by neglecting the dynamic part. When he numerically evolves the system with sufficiently refined spatial discretization so that the continuum limit is well resolved, he gets only periodic secuences, while if the spatial discretization is made too coarse, so that the system is "inherently discrete", he observes nonperiodic complex sequences. He goes on to suggest that this property may be true of all the self-organizing models as well: the complexity they produce may be a result. of their inherent discreteness, and that models with a well defined continuum limit will not give complex nonperiodic sexuences. This issue is fundamental to the fixed irregularity versus self-organization debate, since if the self-organized models repuire a discontinuous nonuniform partitioning of space to generate sufficient complexity, then they too ultimately rest on fixed irregularities.

In this paper, I present a counterexample to Rice's suggestion. A spatially homogeneous model with a well defined continuum limit, is shown to give a complex nonperiodic sequence of events, with a distribution of sizes of events that is independent of the spatial discretization. Previous work, by Horowitz and Ruina [1989] found evidence that complex nomperiodic slip modes could develop in continuum fault models with a number of unstable modes." Because of the numerical costliness of their model, however, they were not able to study the self-organized complexity that can arise in those models: Here, I present an example of a continuum fault model which dynamically generates self-organized complexity. The particular form of complexity produced shows, generically, a power law distribution of small events, and a peak of large events, with both features being seen for two very different types of small scale cutoffs.

The model used here differs in three important ways from the model Rice used: the dimensionality of the space used here is smaller, fully dynamic interactions are solved here as opposed to quasistatic interactions used by Rice, and, finally, the constitutive laws relating friction to motion on the fault are different. Which of these differences may be crucial to the different answers obtained-complexity or not- and, more importantly, which is relevant for earthquakes? The reduced spatial dimension used here is a significant alteration, and it is essential that higher spatial dimensional extensions of this model continue to show complexity if the results are to be relevant to earthquakes. This question is as yet unanswered, and is a topic of current research. The simplified temporal dynamics used by Rice is also a severe alteration with respect to true elastodynamics; by the same 
token, extensions of Rice's model which include complete temporal dynamics are also crucial to its relevance to earthquakes. The final difference, in the constitutive law, is a complex issue that, despite efforts in the laboratory, has not been resolved. We really do not know what effective constitutive equations are relevant at the slip rates, pressures, and temperatures where earthquake occur in the presence of gouge, fluids, and fractured surrounding rocks. Rice has chosen to use constitutive laws obtained from laboratory measurements of materials at relatively low slip rates [Dieterich, 1979]. There is, however, a fundamental problem that arises if one tries to directly apply laboratory derived constitutive relations to earthquake settings, and that is that so much heat would be generated from frictional sliding that rocks would melt [McKenzine and Brune, 1972]. As this is rarely seen [Sibson, 1975], and would, in any case, substantially alter the constitutive equations, some other effect must be going on. The e are a number of possibilities, including ones involving pore fluids [Sibson, 1973] and ones involving new modes of rupture [Shallamach, 1971; Brune, 1994], any of which could substantially alter the constitutive equations that represent the effective friction in an earthquake. In the absence of a sufficient understanding of what constitutive relations do apply to real earthquakes, the best any of us can do is discuss what classes of relations have what effects. I have made a different choice than Rice, choosing to look at the simplest constitutive relations; in this case, that means no history dependence. Despite all these differences, and the unanswered questions relating the work to real earthquake, this work does address one important point concerning the role of discreteness in dynamical models: geometrical irregularities or "inherent discreteness" is not a necessary condition for producing complex nonperiodic sequences.

\section{The Model}

The model is a partial differential equation representing the evolution of slip $S$ along a fault [Myers and Langer, 1993]

$$
\frac{\partial^{2} S}{\partial^{2} t}=\frac{\partial^{2} S}{\partial x^{2}}-S+\nu t-\phi\left(\frac{\partial S}{\partial t}\right)+\eta \frac{\partial^{3} S}{\partial x^{2} \partial t}
$$

where $t$ is time and $x$ is space. This is Newton's equation in dimensionless form for the acceleration of the slip $S$ subject to four forces: a compressional stress $\partial^{2} S / \partial x^{2}$, a shear stress $\nu t-S$, where $\nu$ is the fault loading rate, a nonlinear friction $\phi$ which is a function of the velocity, and a viscous force of strength $\eta$. All the complexity arises from a dynamical instability associated with $\phi$, which is a stick-slip velocity-weakening friction. By stick-slip, we mean the friction resists sliding up to a threshold force; once this threshold is exceeded, sliding occurs. By velocity weakening, we mean that the friction gets smaller as the velocity gets bigger, for some range of velocities. When the friction is velocity strengthening when evaluated at the slip rate $\nu$, the solution $\partial S / \partial t=\nu$ is stable. When it is weakening there, however, this solution is unstable, and a nonconstant motion ensues.

The particular form of $\phi$ used here is:

$$
\phi\left(\frac{\partial S}{\partial t}\right)=\left\{\begin{array}{ll}
(-\infty, 1], & \frac{\partial S}{\partial t}=0 ; \\
\frac{1-\sigma}{1+\frac{\alpha}{1-\alpha} \frac{\sigma}{\partial t}}, & \frac{\partial S}{\partial t}>0 .
\end{array} .\right.
$$

While the total forces on the fault remain less than the threshold force of 1 , the fault remains stuck with $\partial S / \partial t=0$, and is slowly loaded at rate $\nu \ll 1$. When the threshold force is exceeded, the fault begins to slide with initial acceleration $\sigma$. As the velocity initially increases, the friction decreases with the velocity at a rate $\alpha$. The velocity weakening is a crucial ingredient; a linear stability analysis shows that all Fourier modes with wavelengths larger than $2 \pi \sqrt{\eta / \alpha}$ are unstable when sliding in the velocity weakening regime.

The velocity weakening also causes pulses of slip to sharpen into shock fronts. The dynamics then becomes sensitive to the small lengthscale in the problem. The new term in this equation is the viscosity $\eta \partial^{3} S / \partial x^{2} \partial t$, which has been added to give the equation a small lengthscale which then allows a well defined continuum limit [Myers and Langer, 1993; Langer and Nakanishi, 1993]. Together, the friction $\phi$ and the viscosity define the constitutive laws used here to relate dissipation on the fault to motions on it.

To solve this equation numerically, a finite difference approximation of the spatial derivatives are used:

$$
\begin{aligned}
\frac{\partial^{2} S_{j}}{\partial^{2} t}=\frac{1}{a^{2}} & {\left[S_{j+1}-2 S_{j}+S_{j-1}\right]-S_{j}+\nu t } \\
& \quad \phi\left(\frac{\partial S_{j}}{\partial t}\right)+\frac{\eta}{a^{2}}\left[\frac{\partial S_{j+1}}{\partial t}-2 \frac{\partial S_{j}}{\partial t}+\frac{\partial S_{j-1}}{\partial t}\right]
\end{aligned}
$$

where $j$ is the label of the $j^{\text {th }}$ element, and the parameter a represents the degree of refinement of the lattice, with the small cutoff lengthscale being $a$, and $a \rightarrow 0$ being the continuum limit. This discretized equation without the viscous term, setting $\eta=0$, is the classic Burridge-Knopoff model [Burridge and Knopoff, 1967]. Previous work with $\eta=0$ found that complex sequences of events were observed with a power law distribution of small events and a peak of large events above the extrapolated small event distribution [Carlson and Langer, 1989]. This distribution was seen to be sensitive to the spatial discretization, however, with both the small and large events scaling with a [Carlson, Langer, Shaw, and Tang, 1991]. The main result of this paper is the demonstration that the addition of the viscous term $\eta \partial^{3} S / \partial x^{2} \partial t$, which introduces a small lengthscale

$$
\epsilon=\pi \sqrt{\eta / \alpha}
$$

below which the equation is stable, displays complex behavior that is essentially independent of $a$, for $a$ small enough compared to $\epsilon$. The small parameter $\eta$ replaces the small length $a$ as being relevant in setting the lower cutof of the small event power law region, and the upper cutoff of the size of the largest events. For these two very different types of small scale cutoffs, the distribution of sizes of events looks very similar. This result points out the universality (i.e. insensitivity) of the complexity produced with respect to the details of the physics of the small scale cutoff.

\section{Results}

Beginning from any nonsmooth initial condition, the system evolves within a few loading cycles to a statistically steady state, with a broad distribution of sizes of events. As in the case of $\eta=0$, there are two different types of behavior displayed by the model. When $\alpha \lesssim 1$, there are only small events which scale with $\sigma$. In contrast, when $\alpha \gtrsim 1$, there are small events that scale with $\sigma$ and large events that scale with 1 . While $\alpha$ is a crucial parameter in the problem, the value that best corresponds with the Earth is very uncertain. Fortunately, from the point of view of the model, the important question is only whether $\alpha$ is small or large compared to 1 ; the two different types of behavior depend on whether $\alpha$ is small or large, but the behavior is otherwise relatively insensitive to the exact value of $\alpha$, with the velocity-weakening friction we examine here. Since the large $\alpha$ case is somewhat more robust and interesting, producing both small and large events, we will focus our attention on it in this paper.

The size of an event is given by the moment, which is the sum of slips $\delta S$ of all the blocks that moved during an event:

$$
M=a \sum_{j} \delta S_{j}
$$

where the factor a comes in because each element is size $a$. The magnitude of an event is just the logarithm of the moment

$$
\mu=\ln M
$$

In Figmres 1-3 the rate $R$ of events (per unit fault length per unit loading time) having magnitudes between $\mu$ and $\mu+\delta \mu$ is plotted. The friction parameters $\alpha$ and $\sigma$ are held fixed, with $\alpha=6$ and $\sigma=.01 \mathrm{in}$ all the figures, while $a$ and $\eta$ are varied. The results shown are generic for a broad range of $\alpha$ and $\sigma$, holding for $\sigma$ small $(\sigma<1)$ and $\alpha$ large $(\alpha \gtrsim 2)$. Periodic boundary conditions are 


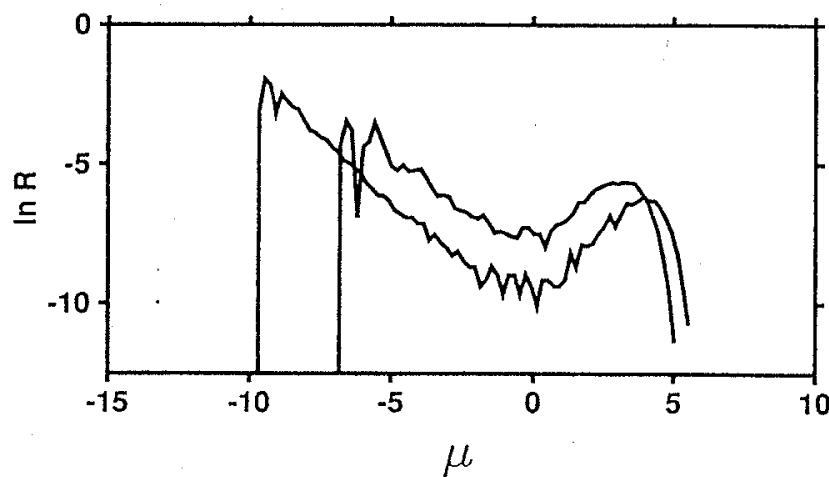

Fig. 1. The distribution of sizes of events for the small lengthscale being $a$. The differential rate of events $R(\mu)$ having magnitudes between $\mu$ and $\mu+d \mu$ is plotted. The rate is expressed as the number of events per unit fault loading per unit fault length. The two different curves differ only in the value of $a$, having the same parameters $\alpha=6, \sigma=.01$, and $\eta=0$. The top curve has $a=.25$ while the bottom curve has $a=.125$. Each curve has two different types of events. The small events show a power law in the rate versus moment, seen in the straight line on the $\log R$ versus magnitude $(\log M)$ plot. The large events occur at a rate that is larger than the rate that would be obtained by extrapolating from the small event rate. Note that the distribution continues to change as a changes.

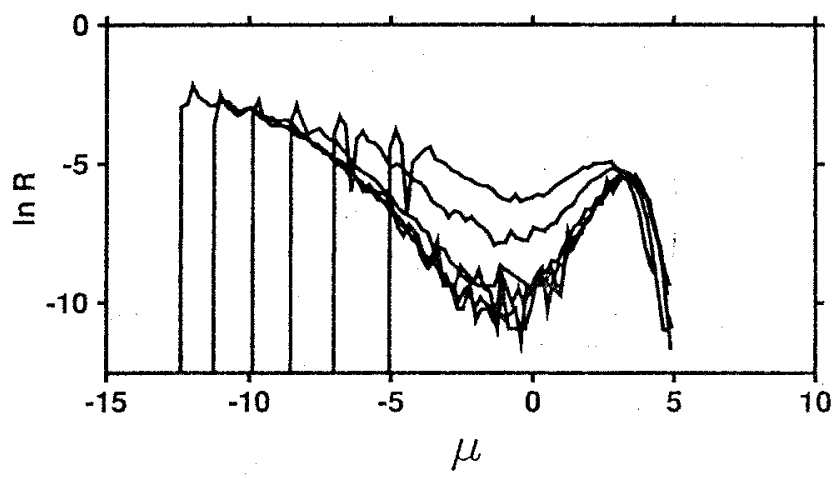

Fig. 2. The distribution of sizes of events, for the distribution going from depending on a to depending on $\eta$. to $c$. As in Figure 1 , the rate of events $R$ versus magnitude $\mu$ is plotted. The different curves are for fixed $\alpha=6, \sigma=.01$, a fixed nonzero $\eta=.02$, and differing a. These values of $\eta$ and $\alpha$ correspond to a value of $\epsilon=.25$. The values of $a$ shown are decreased by a constant factor of $\sqrt{2}$, with $a=$ $.353, .250, .176, .125, .088$, and .062 plotted. Note the transition from a distribution that depends on $a$, to a limiting distribution that collapses onto a curve that is independent of $a$. This transition occurs at around $a=.15$, which is of order $\epsilon$.

used. The system size is $N$, but as long as $N$ is large enough, and $\nu \ll 1 / N$, the largest events will be smaller than the system size, and $N$ does not enter into the problem. In Figure $1, \eta=0$, while $a$ is varied. The change in the distribution as a function of the small lengthscale $a$ can be seen. Note that while the smallest and largest events scale with a [Carlson, Langer, Shaw, and Tang, 1991], the magnitude where the transition between the power law small events and peak of large events occurs is independent of $a$, and the exponent of the power law distribution of small events is also independent of $a$ [Carlson and Langer, 1989].

In Figure $2, \eta=.02$, and again $a$ is varied. Here, we can see the crossover as $a$ becomes small enough and the distribution becomes independent of $a$. When $a$ becomes small enough, all the curves collapse onto the same distribution. The only difference between the curves is the cutoff of the very smallest events, which scale as $\sigma a^{3}$, with the $a \rightarrow 0$ limit being well defined. Finally, in Figure 3, two

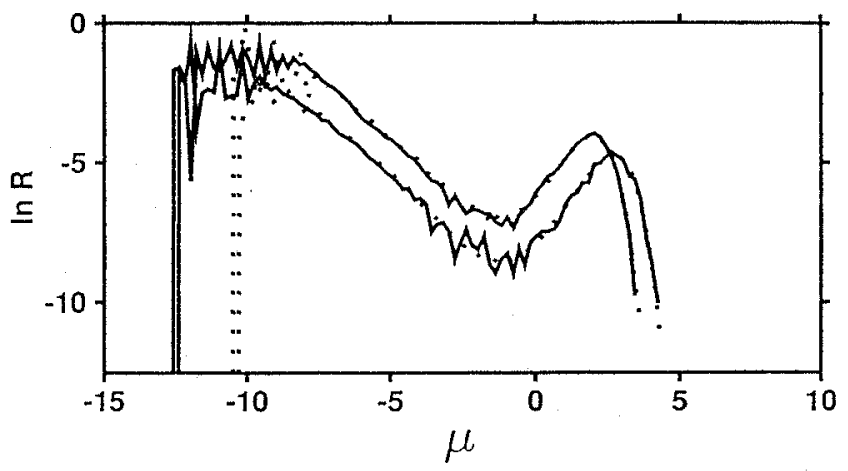

Fig. 3. The distribution of sizes of events for the distribution depending on $\eta$, for two different values of $\eta$. As in Figures 1 and 2, $R(\mu)$ is plotted for fixed $\alpha=6, \sigma=.01$. Here, two different values of $\eta$ are shown: $\eta=.25$ and $\eta=.0625$. Two different values of $a$ are used for each value of $\eta$. Since $a$ is small enough the curves having the same $\eta$, but different $a$, collapse onto each other. The two solid curves have $a=.0625$ and two different values of $\eta$, with $\eta=.25$ and $\eta=.0625$. The are also two dashed curves, which have $a=.25$ and the same two $\eta$ values as the solid curves, $\eta=.25$ and $\eta=.0625$; since the dashed curves overlay the solid curves so well, they are difficult to see. Note the similarity of these distributions, which depend on $\eta$, as compared to the distributions in Figure 1 which depend on a- two very different types of small scale cutoff. Note also the similarity of the change in the distribution with $\epsilon$ seen here, as $\epsilon$ is changred by a factor of 2 , compared to the change in the distribution in Figure 1 when $a$ is changed by a factor of 2 .

different values of $\eta$ are used, $\eta=.25$ and $\eta=.0625$, along with two different $a$ 's, which are small enough so they collapse onto the continuum limit distributions. Here, the curves having different values of $a$ but the same value of $\eta$ collapse onto the same distribution, while the two different values of $\eta$ yield different distributions. Note that the change in the distribution when the small lengthscale is changed by a factor of 2 (corresponding to the change in $\eta$ of a factor of 4 ) in Figure 3 looks very similar to the change in the distribution under a factor of 2 change in the small lengthscale in Figure 1.

\section{Conclusion}

What is the relevance of the complexity produced by this model with respect to real earthquakes? Two big questions arise when we try to answer this one. First, the model used only 1 dimensional elastic interactions, which are all short range, while the higher dimensional elasticity of real faults produces interactions between distant parts of a fault. Whether or not higher dimensional extensions of the model continue to produce complexity is the key open question, a concern also raised by Rice [1993] (the 2 dimensional case will be sufficient to answer the question, and is an active area of current research). The second set of questions, assuming that the higher dimensional models do continue to produce complexity, is what types of constitutive laws produce what types of complexity, and how we might distinguish between different constitutive laws, whether through the types of complexity produced, through laboratory measurements incorporating the full range of relevant physical processes occurring in earthquake settings, or through a derivation of a constitutive law from physical processes.

In this paper we have seen that, a spatially uniform self-organizing model with a well defined continum limit can produce complex nonperiodic behavior. This provides a counterexample to the conjecture of Rice [1993] that, "inherent discreteness" might be an essential inbredient in allowing the self-organizing models to produce complexity. An additional result was that the culalitative features of the complexity produced by the model were invariant with respect to 
t.wo very different types of small scale cutoffs, suggesting a universality of the complexity with respect. to the details of the small scale cutoff.

Acknowledgements. I am deeply grateful to Jim Langer for both introducing the viscous term and pointing out that it ought to provide a good continuum limit. I also thank Jean Carlson for numerous discussions, and for sharing her initial work on this problem. This work was supported by USGS grant 1434-93-G-2284 and by SCEC grant USC-572726.

\section{References}

Bak, P., and C. Tang, Earthquakes as a self-organized critical phenomena, J. Geo. Res., 94, 15635, 1989.

Bak, P., C. Tang, and K. Wiesenfeld, Self-organized criticality: an explanation of $1 / \mathrm{f}$ noise, Phys. Rev. Lett., 59, 381-384, 1987.

Brune, unpublished, 1994

Burridge, R., and L. Knopoff, Model and theoretical seismicity, Bull. Seismol. Soc. Am., 57, 3411-3471, 1067.

Carison, J.M. and J.S. Langer, Mechanical model of an earthquake fault, Phys. Rev. A, 40,6470, 19896 .

Carlson, J.M., J.S. Langer, B.E. Shaw, and C. Tang, Intrinsic properties of a Burridge-Knopoff model of an earthquake fault, Pliys. Rev. A, 44, 884-897, 1991:

Cowie, P.A., C. Vanneste, and D. Sornette, Statistical physics model for the spatio-temporal evolution of faults: preprint, 1993.

Dieterich, J.H., Modeling of rock friction: 1. Experimental results and constitutive equations, J. Geo. Res., 84, 2161, 1979.
Horowitz, F.G. and A. Ruina, Slip patterns in a spatially homogeneous fault model, J. Geo. Res., 94, 10279, 1989

Langer, J.S., and H. Nakanishi, Models of crack propagation II: two dimensional model with dissipation on the fracture surface, preprint, 1993.

McKenzine, D.P., and J.N. Brune, Melting on fault planes during large earthquakes, Geophys. J. Roy. Astron. Soc., 29, 65, 1972.

Myers, C.R. and J.S. Langer, Rupture propagation, dynamical front selection, and the role of small length scales in a model of an earthquake fault, Phys. Rev. E, $\{7,30481993$

Rice, J.R., Spatio-temporal complexity of slip on a fault, J. Geo. Res., $98,9885,1993$

Shallamach, A., How does rubber slide? Wear, 17, 301, 1971.

Sibson, R.H., Interactions Between Temperature and Pore Fluid Pressure During Earthquake Faulting and a Mechanism for Partial or Total Stress Relief, Nature, 243, 66, 1973.

Sibson, R.H., Generation of Pseudotachylyte by ancient seismic faulting, Geophys. J. Roy. Astron. Soc., 43, 775, 1975.

$\mathrm{Xu}, \mathrm{H}-\mathrm{J}, \mathrm{B}$. Bergersen, and K. Chen, A new crack propagation model of earthquakes, J. Pliys. A, 25, L1251, 1992.

Shaw, B.E. Lamont-Doherty Earth Observatory, Columbia University, Palisades NY, 10964

(Received: November 19, 1993; Revised: March 10, 1994; Accepted: Jane 10, 1994.) 